\title{
SELEÇÃO DE DESCRITORES VEGETATIVOS PARA CARACTERIZAÇÃO DE ACESSOS DE GUARIROBA (Syagrus oleracea (Mart.) Becc.) $^{1}$
}

\author{
JEFFERSON FERNANDO NAVES PINTO ${ }^{2}$, EDESIO FIALHO DOS REIS ${ }^{3}$ \\ FABIO GELAPE FALEIRO ${ }^{4}$, ELAINE CRISTINA CASTELHANO BARBOSA ${ }^{5}$ \\ HELLEN FERNANDA NUNES ${ }^{6}$ JEEDER FERNANDO NAVES PINTO $^{7}$
}

RESUMO - O presente trabalho teve como objetivo selecionar descritores morfológicos vegetativos e determinar sua importância relativa na caracterização de acessos de guariroba, assim como verificar a associação entre os descritores descartados e os demais. Foram avaliados 18 descritores morfológicos, sendo 8 relativos à folha e 10 ao estipe, em 36 acessos de guariroba do Estado de Goiás. Os dados foram submetidos à análise de variáveis canônicas, e o descarte dos descritores foi realizado tendo como base no autovalor, o qual não excedeu 0,70 . Foram descartados $55 \%$ dos descritores, sendo 5 relativos à folha e 5 ao estipe. O descarte destes descritores não ocasionou perda de informações, por apresentaram correlações significativas com os descritores selecionados. A contribuição relativa dos descritores selecionados foi semelhante para A7A, ENI7180, DFBF, D0, CPC e DPC, sendo os descritores ENI70 e ENS70 os que apresentaram as menores contribuições.

Termos para indexação: variabilidade genética, análise multivariada, variáveis canônicas, gueroba.

\section{VEGETATIVE DESCRIBERS SELECTION FOR ACCESSES CHARACTERIZATION OF SYAGRUS OLERACEA PALM}

\begin{abstract}
Summary - The present paper had the purpose of selecting morphological vegetative descriptors and to determine their relative importance in the characterization of guariroba accesses, as well as to verify the association between discarded and accepted descriptors.. Eighteen morphological descriptors, of which 8 relative to the leaf and 10 to the stipe, in 36 guariroba accessions in the State of Goiás were evaluated. The discarded descriptors did not cause loss of information, as they presented significant correlations with the descriptors that were accepted. The relative contribution of the selected descriptors was similar for A7A, ENI7180, DFBF, D0, CPC and DPC, with descriptors ENI70 and ENS70 having the lowest contribution. Index Terms: genetic variability, multivariate analysis, canonical variables, gueroba.
\end{abstract}

\section{INTRODUÇÃO}

A única palmeira que possui palmito de sabor amargo entre as exploradas comercialmente no Brasil, é a guariroba (Syagrus oleracea (Mart.) Becc.). Essa espécie é nativa do Cerrado e é responsável por boa parte do palmito consumido nesta região.

A guariroba é uma espécie incipientemente domesticada (CLEMENT, 2001) e, por isso, informações sobre a caracterização morfológica de acessos que possam ser utilizados em programas de melhoramento, são escassas, além de conhecimento sobre características importantes na discriminação da variabilidade existente entre acessos.

Um dos principais motivos que reduzem a expansão da cadeia produtiva da guariroba, segundo Nascente (2003), é a grande desuniformidade no desenvolvimento da planta, o que acarreta em maior tempo de colheita, refletindo em maior espaço de tempo na ocupação do solo, contribuindo para menor atração de produtores à cultura. Outro motivo é a variabilidade no sabor amargo do palmito, o qual, segundo Nunes (2007), vai desde o levemente amargo até o amargo bem acentuado. Isto dificulta a

\footnotetext{
'(Trabalho 161-09). Recebido em: 30-06-2009. Aceito para publicação em: 10-12-2009. Parte da dissertação do primeiro autor. ${ }^{2}$ Professor Adjunto do Departamento de Biologia, Universidade Federal de Goiás/Campus Jataí, Jataí, GO. CEP 75800-000. E-mail: edesio7@brturbo.com.br

${ }^{3}$ Embrapa Cerrados, Caixa Postal 08223, CEP 73301-970 Planaltina, DF. E-mail: ffaleiro@cpac.embrapa.br,

${ }^{4}$ Professor Assistente do Departamento de Biologia, Universidade Federal de Goiás/Campus Jataí, Jataí, GO. CEP 75800-000. E-mail: eccastel@yahoo.com.br

${ }_{5}^{5}$ Discente do Programa de Pós-Graduação em Agronomia, Universidade Federal de Goiás/Campus Jataí, Jataí, GO. CEP 785800-000. E-mail: hllnunes@hotmail.com

${ }^{6}$ Discente do Curso de Agronomia, Universidade Federal de Goiás/Campus Jataí, Jataí, GO. CEP: 75800-000. E-mail: jeedernaves@hotmail.com
} 
padronização do palmito, tornando-o menos atrativo para a indústria de conservas.

A caracterização de plantas perenes, como as palmeiras, tem sido realizada com o auxílio de uma lista de descritores botânicos, morfológicos e agronômicos, os quais muitas vezes são utilizados sem critérios relativos a sua contribuição real para a variabilidade, provocando, assim, aumento de tempo e mão de obra na caracterização das plantas (OLIVEIRA et al., 2006).

$\mathrm{O}$ aumento no número de descritores, na grande maioria das vezes, contribui para melhorar as informações da diversidade genética entre os materiais em estudo. No entanto, o aumento do número de descritores nem sempre melhora a discriminação dos materiais, pois, conforme Daher et al. (1997), se um descritor estiver contribuindo na detecção da variabilidade que outro já discrimina, considera-se que o segundo é redundante em relação ao primeiro, podendo, assim, ser descartado. Dessa maneira, a retirada dos descritores redundantes é vantajosa, por reduzir o trabalho de coleta dos dados e melhorar a interpretação dos mesmos (PEREIRA et al., 1992).

A redução no número de descritores morfológicos é relatada por outros pesquisadores, os quais utilizaram diversas técnicas de descarte, com o intuito de otimizar o trabalho de coleta dos dados (BALAKRISHNAN; NAMBOODIRI, 1987; RIBEIRO, 1993; MARTEL et al., 2003; OLIVEIRA 2005). Segundo Balakrishnan e Namboodiri (1987) e Ribeiro et al. (1999), para o coqueiro (Cocos nucifera L.), é necessário utilizar somente três ou quatro descritores morfológicos; para o açaí (Euterpe oleracea Mart.), usam-se apenas quatro descritores (Oliveira, 2005), e para a pupunha (Bactris gasipaes Kunth), é necessário utilizar 10 descritores morfológicos para discriminar as raças desta espécie (Martel et al., 2003).

O objetivo deste trabalho foi selecionar descritores morfológicos vegetativos e determinar sua importância relativa na caracterização de acessos de guariroba, assim como verificar a associação entre os descritores descartados e os aceitos.

\section{MATERIAL E MÉTODOS}

Para a realização deste trabalho, foram analisados 36 acessos de guariroba obtidos em 36 pontos de coleta de quatro regiões do Estado de Goiás (Jataí, Aurilândia, Aragoiania e Catalão). Os pontos de coleta foram estabelecidos de forma aleatória, considerando os locais de ocorrência da espécie. Dentro de cada município, foram avaliadas três propriedades escolhidas ao acaso e, dentro das mesmas, três plantas avaliadas também de forma casualizada. As três plantas dentro da propriedade foram consideradas como repetição.

Nestes acessos, foram avaliados 18 descritores morfológicos, sendo 8 relativos à folha e 10 ao estipe. As avaliações foram realizadas em plantas com, no mínimo, seis metros de altura, e as medidas foliares foram feitas em folhas completamente desenvolvidas. Os seguintes caracteres foram avaliados: número de folhas vivas (NFV), número de pinas do lado direito da raque (NPLD), número de pinas do lado esquerdo da raque (NPLE), comprimento da folha (CF), diâmetro central da Folha (DCF), diâmetro final da bainha foliar (DFBF), comprimento Da pina central da folha (CPC), diâmetro da pina central da folha (DPC), diâmetro do estipe a $0 \mathrm{~cm}$ do solo (D0), diâmetro do estipe a $50 \mathrm{~cm}$ do solo (D50), diâmetro do estipe a $100 \mathrm{~cm}$ do solo (D100), altura do $7^{\circ}$ anel, partindo-se da base da planta (A7A), espessura do $7^{\circ}$ anel na sua região menos espessa (E70), espessura do $7^{\circ}$ anel, a $180^{\circ}$ da região menos espessa (E7180), espessura do nó superior ao ponto menos espesso do $7^{\circ}$ anel (ENS70), espessura do nó superior ao $7^{\circ}$ anel, a $180^{\circ}$ de sua menor espessura (ENS7180), espessura do nó inferior ao ponto menos espesso do $7^{\circ}$ anel (ENI70) e espessura do nó inferior ao $7^{\circ}$ anel a $180^{\circ}$ de sua menor espessura (ENI7180).

Os dados destes descritores foram inicialmente submetidos à análise de variância e, em seguida, realizou-se a análise multivariada mediante as variáveis canônicas, conforme Cruz (2006), com base nos 18 descritores morfológicos avaliados.

Foi realizada a identificação dos descritores redundantes, por meio da metodologia proposta por Jollife (1973), sendo indicado para descarte todo descritor que apresentou maior coeficiente de ponderação em valor absoluto (autovetor), na variável canônica de autovalor menor, partindo-se da última variável até aquela que seu autovalor não excedeu 0,70. Para auxiliar na decisão de descarte, foram estimados os coeficientes de correlação de Pearson entre todos os descritores, para verificar a associação entre os descritores descartados e os remanescentes. A significância do coeficiente de correlação foi verificada pelo teste de $t$.

Após o descarte dos descritores redundantes, procedeu-se a análise multivariada com os descritores selecionados, para se obterem as novas variáveis canônicas.

Todas as análises estatísticas foram realizadas com o auxilio do Programa Genes (Cruz, 2006). 


\section{RESULTADOS E DISCUSSÃO}

Pela análise de variância univariada, houve diferenças significativas entre os acessos, $\operatorname{com} p<0,01$ e $\mathrm{p}<0,05$ para dez e dois descritores morfológicos, respectivamente, sendo que em apenas seis descritores não houve significância (Tabela 1). Os coeficientes de variação experimental estão entre 8,84 para NPLDF e 42,95 para ENS70. Pode-se verificar que os maiores coeficientes de variação estão associados às características que não apresentaram diferenças significativas pelo teste $\mathrm{F}$, evidenciando que, para elas, as variações ao acaso foram mais evidentes.

Em estudos de divergência genética utilizando a metodologia das variáveis canônicas, tem-se o propósito de identificar acessos similares por meio de ferramentas gráficas (gráficos de dispersão bi ou tridimensionais), que possibilitam simplificar a interpretação dos resultados (NEGREIROS et al., 2008). Dessa forma, é desejável que a maior parte da variância esteja acumulada nas duas primeiras variáveis canônicas (CRUZ et al., 2004).

No presente estudo, pode-se verificar que a variância acumulada entre as 18 variáveis não demonstrou distribuição concentrada nas primeiras variáveis, podendo-se constatar que somente a partir da sexta variável canônica foi possível acumular uma porcentagem satisfatória, com $71,36 \%$ da variabilidade total dos acessos (Tabela 2). As três primeiras variáveis canônicas explicam $45,31 \%$ da variação total, com a primeira variável explicando $18,39 \%$, a segunda $15,47 \%$ e a terceira somente $11,45 \%$.

Segundo Pereira et al. (1992), a distribuição da variância está associada ao número de descritores utilizados na análise, estando concentrada nas primeiras variáveis canônicas, apenas quando se utiliza um número reduzido de descritores de interesse agronômico ou que estejam em um mesmo grupo (folha, floração, frutos, etc.). Salienta-se, ainda, que quando vários descritores são redundantes, ou seja, explicam a mesma variação, há tendência de concentração da explicação nas primeiras variáveis canônicas. A análise de variáveis canônicas permite realizar o descarte de descritores morfológicos, eliminando, desta forma, aqueles que oferecem pouca importância (contribuição) no estudo de divergência (NEGREIROS et al., 2008).

Utilizando-se da metodologia proposta por Jolliffe (1973), que estabelece a eliminação dos descritores de maior peso nas variáveis canônicas cuja associação entre autovetores e autovalores são inferiores a 0,7 , foram descartados dez descritores morfológicos, sendo cinco relacionados à morfologia da folha (NFV, NPLDF, NPLEF, CF e DCF) e cinco relacionados à morfologia do estipe (D50, D100, E70, E7180 e ENS7180). Essas exclusões indicam que $62,5 \%$ dos descritores morfológicos relacionados à morfologia da folha são desnecessários para a caracterização dos acessos de guariroba. Em relação aos descritores do estipe, 50\% são redundantes. Este descarte traz uma redução de $55 \%$ dos descritores morfológicos avaliados, o que facilita o processo de avaliação. Percentuais de descarte próximos a estes foram encontrados por Pereira (1989), Daher (1993), Cruz (1990), Cury (1993), Strapasson (1997), Martel et al.(2003) e Oliveira et al.(2006).

As estimativas da correlação de Pearson, entre o conjunto de descritores redundantes e o dos selecionados, demonstram que o descarte não revelou perda considerável de informação, pois os descritores eliminados apresentam, no mínimo, três correlações significativas com os descritores selecionados (Tabela 3). Isso indica que é possível a eliminação de descritores sem perda de informação, pois os mesmos estão correlacionados a outros que permaneceram na análise.

Após o descarte, na nova análise com os oito descritores remanescentes, pode-se constatar (Tabela 4) que as quatro primeiras variáveis canônicas explicam cerca de $76,845 \%$ da variabilidade morfológica contida na guariroba, sendo $26,765 \%$, $21,548 \%, 15,496 \%$ e $13,037 \%$, respectivamente. Isso leva a um ganho adicional de $20,91 \%$ em relação às obtidas anteriormente, com base nos 18 descritores morfológicos avaliados (Tabela 2). Percentuais próximos aos obtidos neste estudo foram registrados em análise por componentes principais por Daher et al. (1997), Strapasson et al. (2000), Crochemore et al. (2003), Oliveira et al. (2006), Gomes (2007) e Karia (2008). Estes resultados justificam a utilização desta metodologia, por proporcionar uma simplificação na estrutura dos dados analisados (KARIA, 2008; NEGREIROS, et al. 2008).

Com base na contribuição relativa dos descritores morfológicos remanescentes (Ttabela 5), utilizando-se do critério proposto por Singh (1981), pode-se verificar que, para os acessos avaliados, tem-se que, dos 8 descritores selecionados, A7A, ENI7180, DFBF, D0, CPC e DPC contribuíram de forma semelhante para a divergência entre os acessos, e os descritores ENI70 e ENS70 apresentaram menores contribuições. Desta forma, pode-se afirmar que os descritores selecionados são importantes na caracterização dos acessos de guariroba por apresentar contribuições importantes na discriminação da divergência. 
Na análise da dispersão dos escores das três primeiras variáveis canônicas (Figura 1), nota-se que, com a contribuição das três primeiras variáveis com $63,8 \%$ da variação total, é possível verificar a divergência entre vários acessos em estudo.

Tais resultados indicaram que o método de descarte das variáveis foi eficiente na identificação e eliminação de variáveis redundantes. Forneceram, ainda, informações para considerar que todos os descritores selecionados são importantes no estudo de caracterização da divergência genética entre os acessos de guariroba. Estes descritores devem compor a lista de descritores mínimos para a espécie, a qual deve ser complementada por aqueles relacionados com a produção e com as características organolépticas do palmito.

TABELA 1 - Resumo das análises de variância, obtidas para os 18 descritores morfológicos avaliados em 36 acessos de guariroba, no Estado de Goiás.

\begin{tabular}{cccc}
\hline \multirow{2}{*}{ FV } & \multicolumn{2}{c}{ Quadrado Médio } & \multirow{2}{*}{ CV\% exp. } \\
\cline { 2 - 3 } NFV & Acesso & Resíduo & 20,99 \\
NPLD & $15,46^{* *}$ & 5,52 & 8,84 \\
NPLE & $389,96^{*}$ & 181,387 & 9,52 \\
CF & 362,76 & 205,09 & 14,09 \\
DCF & $0,26^{* *}$ & 0,11 & 29,42 \\
DFBF & 0,15 & 0,11 & 19,43 \\
CPC & $1,46^{* *}$ & 0,56 & 21,23 \\
DPC & $337,06^{* *}$ & 134,81 & 17,69 \\
D0 & $0,587^{* *}$ & 0,243 & 21,95 \\
D50 & $68,23^{* *}$ & 34,21 & 19,12 \\
D100 & 23,54 & 13,52 & 17,56 \\
A7A & $17,01^{*}$ & 8,24 & 25,74 \\
E70 & $635,87^{* *}$ & 255,42 & 31,55 \\
E7180 & $19,31^{* *}$ & 6,88 & 29,77 \\
ENS70 & $19,79^{* *}$ & 8,09 & 42,95 \\
ENS7180 & 0,223 & 0,199 & 40,09 \\
ENI70 & 0,74 & 0,62 & 38,46 \\
ENI7180 & 0,195 & 0,157 & 30,32 \\
\hline
\end{tabular}

* e ** significativo pelo teste $\mathrm{F}$ ao nível de 5 e $1 \%$ de probabilidade, respectivamente.

(NFV) número de folhas vivas, (NPLD) número de pinas do lado direito da raque, (NPLE) número de pinas do lado esquerdo da raque, (CF) Comprimento da folha, (DCF) Diâmetro central da folha, (DFBF) Diâmetro final da bainha foliar, (CPC) Comprimento da pina central, (DPC) Diâmetro da pina central, (D0) Diâmetro do estipe a 0cm, (D50) Diâmetro do estipe a 50cm, (D100) Diâmetro do estipe a $100 \mathrm{~cm},(\mathrm{~A} 7 \mathrm{~A})$ Altura do $7^{\circ}$ anel partindo-se de $0 \mathrm{~cm}$, (E70)Espessura do $7^{\circ}$ anel a $0^{\circ}$, (E7180) Espessura do $7^{\circ}$ anel a $180^{\circ},($ ENS70) Espessura do nó superior ao $7^{\circ}$ anel a $0^{\circ}$, (ENS7180) Espessura do nó superior ao $7^{\circ}$ anel a $180^{\circ}$, (ENI70) Espessura do nó inferior ao $7^{\circ}$ anel a $0^{\circ}$, e (ENI7180) Espessura do nó inferior ao $7^{\circ}$ anel a $180^{\circ}$. 
TABELA 2 - Autovalores, Variância, Variância Acumulada, obtidas a partir dos dezoito descritores morfológicos avaliados, em 36 acessos de guariroba, no Estado de Goiás.

\begin{tabular}{cccc}
\hline Variáveis Canônicas & Autovalores & Variância (\%) & Variância Acumulada (\%) \\
\hline $\mathbf{1}$ & 2,4176 & 18,39 & 18,39 \\
$\mathbf{2}$ & 2,0338 & 15,47 & 33,86 \\
$\mathbf{3}$ & 1,5052 & 11,45 & 45,31 \\
$\mathbf{4}$ & 1,3954 & 10,61 & 55,93 \\
$\mathbf{5}$ & 1,1749 & 8,94 & 64,86 \\
$\mathbf{6}$ & 0,8538 & 6,50 & 71,36 \\
$\mathbf{7}$ & 0,7508 & 5,71 & 77,07 \\
$\mathbf{8}$ & 0,6373 & 4,85 & 81,92 \\
$\mathbf{9}$ & 0,5826 & 4,43 & 86,35 \\
$\mathbf{1 0}$ & 0,4281 & 3,26 & 89,61 \\
$\mathbf{1 1}$ & 0,3214 & 2,45 & 92,05 \\
$\mathbf{1 2}$ & 0,2616 & 1,99 & 94,04 \\
$\mathbf{1 3}$ & 0,2519 & 1,92 & 95,96 \\
$\mathbf{1 4}$ & 0,1693 & 1,29 & 97,25 \\
$\mathbf{1 5}$ & 0,1287 & 0,98 & 98,22 \\
$\mathbf{1 6}$ & 0,1025 & 0,78 & 99,00 \\
$\mathbf{1 7}$ & 0,0738 & 0,56 & 99,56 \\
$\mathbf{1 8}$ & 0,0572 & 0,44 & 100,00 \\
\hline
\end{tabular}

TABELA 3 - Estimativas dos coeficientes de correlação de Pearson entre os descritores morfológicos selecionados e os descartados, avaliados em 36 acessos de Guariroba, no Estado de Goiás

\begin{tabular}{ccccccccc}
\hline \multirow{2}{*}{ Descartados } & \multicolumn{7}{c}{ Selecionados } \\
\cline { 2 - 9 } & DFBF & CPC & DPC & D0 & A7A & ENS70 & ENS7180 & ENI70 \\
\hline NFV & $-0,165$ & $-0,157$ & $-0,177$ & $-0,198^{*}$ & $-0,116$ & $-0,157$ & 0,062 & $0,205^{*}$ \\
NPLD & $-0,235^{* *}$ & 0,089 & $-0,244^{* *}$ & $-0,085$ & $0,550^{* *}$ & $-0,180^{*}$ & $-0,253^{* *}$ & $-0,168$ \\
NPLE & $-0,067$ & $0,191^{*}$ & $-0,13$ & $-0,158$ & 0,173 & $-0,200^{*}$ & $-0,091$ & $-0,139$ \\
CF & $-0,186^{*}$ & $-0,311^{* *}$ & $-0,238^{* *}$ & 0,097 & $-0,075$ & 0,172 & $-0,076$ & $-0,158$ \\
DCF & $-0,165$ & $-0,217^{*}$ & $-0,051$ & $0,206^{*}$ & $-0,172$ & $-0,227^{*}$ & $-0,103$ & $-0,181^{*}$ \\
D50 & $-0,235^{* *}$ & $-0,066$ & $-0,192^{*}$ & $-0,173$ & $-0,192^{*}$ & 0,042 & $-0,253^{* *}$ & $-0,076$ \\
D100 & 0,11 & $0,214^{*}$ & $-0,321^{* *}$ & $-0,207^{*}$ & $-0,190^{*}$ & 0,162 & $-0,136$ & $-0,161$ \\
E70 & $-0,073$ & $-0,147$ & 0,105 & $0,231^{*}$ & $0,805^{* *}$ & $-0,212^{*}$ & $-0,055$ & 0,133 \\
E7180 & $-0,095$ & $-0,182^{*}$ & $-0,252^{* *}$ & $-0,131$ & $-0,183^{*}$ & $0,830^{* *}$ & $-0,194 *$ & $-0,312^{* *}$ \\
ENI7180 & $-0,064$ & $-0,101$ & $0,595^{* *}$ & $0,192^{*}$ & $-0,263^{* *}$ & $-0,057$ & $-0,187 *$ & $-0,17$ \\
\hline
\end{tabular}

**, * : Significativo a 1 e $5 \%$ de probabilidade, pelo teste $\mathrm{t}$.

(NFV) número de folhas vivas, (NPLD) número de pinas do lado direito da raque, (NPLE) número de pinas do lado esquerdo da raque, (CF) Comprimento da folha, (DCF) Diâmetro central da folha, (DFBF) Diâmetro final da bainha foliar, (CPC) Comprimento da pina central, (DPC) Diâmetro da pina central, (D0) Diâmetro do estipe a 0cm, (D50) Diâmetro do estipe a 50cm, (D100) Diâmetro do estipe a $100 \mathrm{~cm},\left(\right.$ A7A) Altura do $7^{\circ}$ anel partindo-se de $0 \mathrm{~cm},\left(\right.$ E70)Espessura do $7^{\circ}$ anel a $0^{\circ}$, (E7180) Espessura do $7^{\circ}$ anel a $180^{\circ}$, (ENS70) Espessura do nó superior ao $7^{\circ}$ anel a $0^{\circ},\left(\right.$ ENS7180) Espessura do nó superior ao $7^{\circ}$ anel a $180^{\circ}$, (ENI70) Espessura do nó inferior ao $7^{\circ}$ anel a $0^{\circ}$, e (ENI7180) Espessura do nó inferior ao $7^{\circ}$ anel a $180^{\circ}$. 
TABELA 4 - Autovalores, Variância, Variância Acumulada, obtidas a partir dos oito descritores morfológicos selecionados.

\begin{tabular}{cccc}
\hline Variáveis Canônicas & Autovalores & Variância (\%) & Variância Acumulada (\%) \\
\hline $\mathbf{1}$ & 1,452 & 26,765 & 26,765 \\
$\mathbf{2}$ & 1,169 & 21,548 & 48,313 \\
$\mathbf{3}$ & 0,841 & 15,496 & 63,808 \\
$\mathbf{4}$ & 0,707 & 13,037 & 76,845 \\
$\mathbf{5}$ & 0,430 & 7,923 & 84,769 \\
$\mathbf{6}$ & 0,414 & 7,635 & 92,404 \\
$\mathbf{7}$ & 0,235 & 4,339 & 96,743 \\
$\mathbf{8}$ & 0,177 & 3,257 & 100 \\
\hline
\end{tabular}

TABELA 5 - Contribuição relativa dos descritores morfológicos selecionados, avaliados em 36 acessos de guariroba, no Estado de Goiás.

\begin{tabular}{ccc}
\hline Descritor & S.j & Valor em \% \\
\hline A7A & 1074,28 & 15,72 \\
ENI7180 & 1062,33 & 15,54 \\
DFBF & 1012,74 & 14,82 \\
D0 & 969,10 & 14,18 \\
CPC & 910,33 & 13,32 \\
DPC & 867,60 & 12,69 \\
ENI70 & 472,36 & 6,91 \\
ENS70 & 466,94 & 6,83 \\
\hline
\end{tabular}

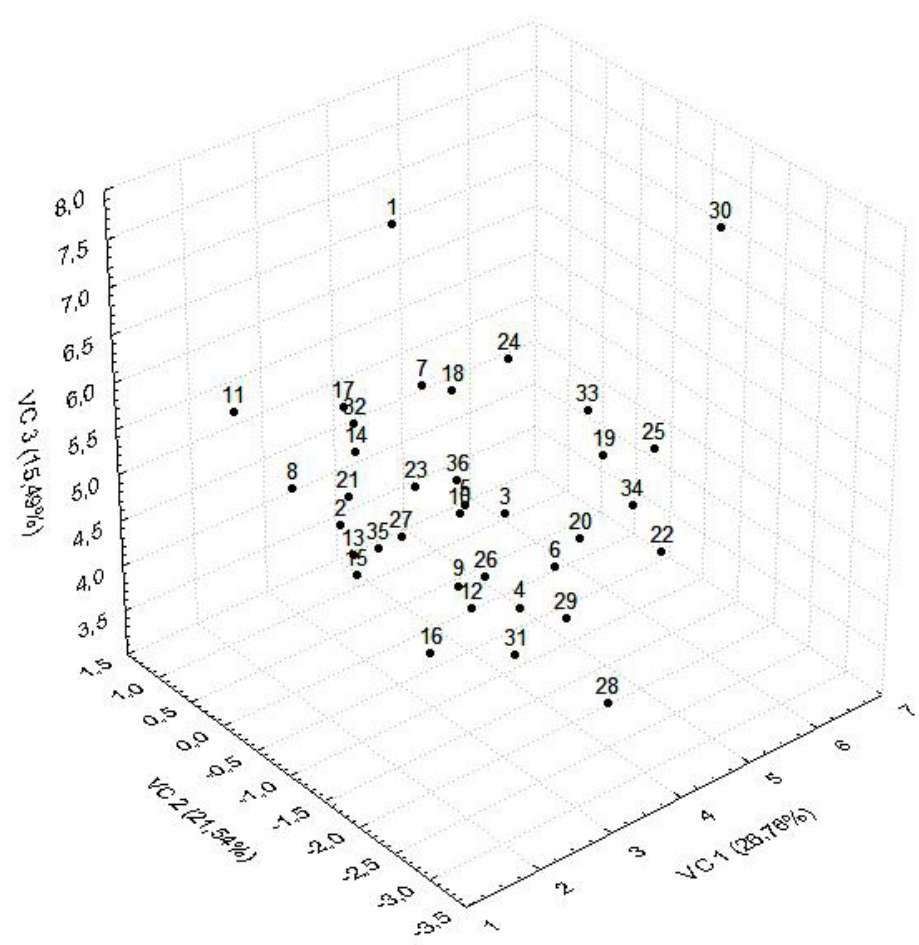

FIGURA 1- Dispersão gráfica em duas dimensões (3D), relativo às três primeiras variáveis canônicas que, juntas, explicam $63,81 \%$ da variação total dos 36 acessos de guariroba. 


\section{CONCLUSÕES}

1-Dos descritores avaliados inicialmente, $45 \%$ mostraram-se importantes na caracterização de acessos de guariroba.

2-O descarte não provocou perda de informação, uma vez que os descritores redundantes estão altamente correlacionados aos descritores remanescentes.

3-Os descritores selecionados são importantes na caracterização dos acessos por apresentarem contribuições importantes na discriminação da divergência.

\section{REFERÊNCIAS}

BALAKRISHNAN, P. C.; NAMBOODIRI, K. M. N. Genetic divergence in coconut. Indian Coconut Journal, Ermakulam, v.18, n.3, p.13-19, 1987.

CLEMENT, C.R. Melhoramento de espécies nativas \{Improvement of native species\}. In: NASS, L.L.; VALOIS, A.C.C.; MELO, I.S.; VALADARESINGLIS, M.C. (Ed.). Recursos genéticos \& melhoramento: plantas. , Rondonópolis: Fundação de Apoio à Pesquisa Agropecuária de Mato Grosso, 2001. p. 423-441.

CROCHEMORE, M. L.; MOLINARI, H. B.; STENZEL, N. M. C. Caracterização agromorfológica do maracujazeiro (Passiflora spp.) Revista Brasileira de Fruticultura, Jaboticabal v. 25, n.1, p. 5-10, 2003.

CURY, R. Dinâmica evolutiva e caracterização de germoplasma de mandioca (Manihot esculenta, Crantz) na agricultura autóctone do sul do Estado de São Paulo. 1993. 103 f. Dissertação (Mestrado) - Escola Superior de Agricultura "Luiz de Queiroz", Universidade de São Paulo, Piracicaba, 1993.

CRUZ, C. D. Aplicação de algumas técnicas multivariadas no melhoramento de plantas. 1990. 188 f. Tese (Doutorado) - Escola Superior de Agricultura "Luiz de Queiroz", Universidade de São Paulo, Piracicaba, 1990.

CRUZ, C. D. Programa genes: análise multivariada e simulação. Viçosa, MG: Ed. UFV, 2006. 175p.
CRUZ, C.D.; REGAZZI, A.J.; CARNEIRO, P.C.S. Modelos biométricos aplicados ao melhoramento genético. Viçosa: Universidade Federal de Viçosa, 2004. v.3, 480 p.

DAHER, R. F. Diversidade morfológica e isoenzimática em capim-elefante (Pennisetumpurpureum Schum.). 1993. 110 f. Dissertação (Mestrado) - Universidade Federal de Viçosa, Viçosa, 1993.

DAHER, R. F.; MORAES, C. F.; CRUZ, C. D. Seleção de caracteres morfológicos em capim-elefante (Pennistum purpureum Schum.). Revista Brasileira de Zootecnia, Brasília, v.26, p.247-259, 1997.

GOMES, D. M. Variabilidade fenotípica de caracteres vegetativos e reprodutivos em população de pupunha (Bactris gasipaes Kunth), 2007. 72 f. Dissertação (Mestrado)-Universidade de Campinas, Campinas, 2007.

JOLLIFFE, I. T. Discarding variables in a principal component analysis. II. Real data. Applied Statistics, London, v.22, p.21-31, 1973.

KARIA, C. T. Caracterização genética e morfoagronômica de germoplasma de Styolosanthes guianenses (Aubl.). 2008. 138 f. Tese (Doutorado) Universidade Federal de Goiás Escola de Agronomia e Engenharia de Alimentos, Goiânia, 2008.

MARTEL, J. H. I., FERRAUDO, A. S.; MOROÔ, J. R.; PERECIN, D. Estatística multivariada na discriminação de raças amazônicas de Pupunheira (Bactris gasipaes Kunth) em Manaus (Brasil). Revista Brasileira de Fruticultura, Jaboticabal, v.25, n.1, p. 115-118, 2003.

NASCENTE, A. S. Caracterização morfológica de progênies nativas de Guariroba (Syagrus oleracea Becc.) no Estado de Goiás. Pesquisa Agropecuária Tropical, Goiânia, v.33, n.2, p. 113-115, 2003.

NEGREIROS, J. S.; ALEXANDRE, R. S.; ÁLVARES, V. S.; BRUCKNER, C. H.; CRUZ, C. D. Divergência genética entre progênies de maracujazeiroamarelo com base em características das plântula. Revista Brasileira de Fruticultura, Jaboticabal, v.30, n.1, p.197-201, 2008 
NUNES, H. F. Associação entre características morfológicas, físicas, químicas e organolépticas do palmito de guariroba. In: FUNAPE/PIBIC. Melhores trabalhos de iniciação Científica / UFG / PRPPG. Goiânia, 2006-2007. p.33-47

OLIVEIRA, M. S. P. Caracterização molecular e morfoagronômica de germoplasma de açaizeiro. 2005. 171f. Tese (Doutorado) - Universidade Federal de Lavras, Lavras, 2005.

OLIVEIRA, M. S. P.; FERREIRA, D. F.; SANTOS, J. B. Seleção de descritores para caracterização de germoplasma de açaizeiro para produção de frutos. Pesquisa Agropecuária Brasileira, Brasília, v.41, n.7, p.1133-1140, 2006.

PEREIRA, V. A. Utilização de análise multivariada na caracterização de germoplasma de mandioca (Manihot esculenta Crantz.). 1989. 180 f. Tese (Doutorado) - Escola Superior de Agricultura Luiz de Queiroz, Universidade de São Paulo, Piracicaba. 1989.

PEREIRA, A.V.; VENCOVSKY, R.; CRUZ, C.D. Selection of botanical and agronomical descriptors for the characterization of cassava (Manihot esculenta Crantz.) germplasm. Revista Brasileira de Genética, Ribeirão Preto, v.15, p.115-124, 1992.
RIBEIRO, F. E. Divergência genética entre populações de coqueiro gigante (Cocos nucifera L.) do Brasil. 1993. 84 f. Dissertação (Mestrado) - Universidade Federal de Lavras, Lavras, 1993.

RIBEIRO, F. E. R.; SOARES, A. R.; RAMALHO, M. A. Divergência genética entre populações de coqueiro-gigante-do-Brasil. Pesquisa Agropecuária Brasileira, Brasília, v.34, n.9, p.1615-1622. 1999.

SINGH, D. The relative importance of characters affecting genetic divergence. The Indian Journal of Genetic and Plant Breeding, New Delhi, v. 41, n. 1, p. 237-245, 1981.

STRAPASSON, E. Seleção de descritores na caracterização de germoplasma de Paspalum através de componentes principais. 1997. 95 f. Dissertação (Mestrado) - Escola Superior de Agricultura Luiz de Queiroz, Universidade de São Paulo, Piracicaba, 1997.

STRAPASSON, E.; VENCOVSKY, R.; BATISTA, L. A. R. Seleção de descritores na caracterização de germoplasma de Paspalum sp. por meio de componentes principais. Revista Brasileira de Zootecnia, Viçosa, MG, v.29, p.373-381, 2000. 